\title{
A new model for imaging tumour progression
}

Pancreatic cancer, which progresses from a premalignant neoplasia to a highly lethal adenocarcinoma, is marked by late-stage clinical presentation and resistance to drug therapy. New findings published in Nature show that Musashi (Msi), a determinant of stem cell fate, is a critical element in pancreatic cancer progression, providing novel targets for therapeutics and opportunities for studying pancreatic tumours.

Msi is a highly conserved RNA-binding protein that is important in maintaining stem cell self-renewal - a key property that is often involved in the progression of cancer from a benign to a malignant disease. The authors of the latest study had previously found that Msi signalling was reactivated in haematologic malignancies, but whether it was also important in pancreatic cancer progression, and whether it might be a viable therapeutic target was unknown.

The investigators first developed a new reporter mouse model, in which Msi-expressing cells within the tumour could be noninvasively imaged to understand their role in cancer progression and drug resistance. "We developed this model in part because we had noticed that this [Msi] signal was broadly expressed in stem and progenitor cells of multiple tissues, and reactivated in aggressive cancers," explains author Tannishtha Reya. "Thus, this model could be a powerful tool for tracking the behaviour and potential of stem cells within normal and malignant tissues."

The model showed that Msi expression increased with pancreatic cancer progression, and that cancer growth, drug resistance and lethality were driven by Msi-positive cells. Genetic deletion of Msi in a mouse model of pancreatic cancer was found to improve survival, which led the researchers to develop antisense oligonucleotide (ASO) inhibitors against Msi. These inhibitors effectively blocked Msi-expression, halting tumour growth in animal models as well as patient-derived cancer cells.

"We are excited about the potential power of the new reporter model we have developed," says Reya, adding that this model could serve as an effective

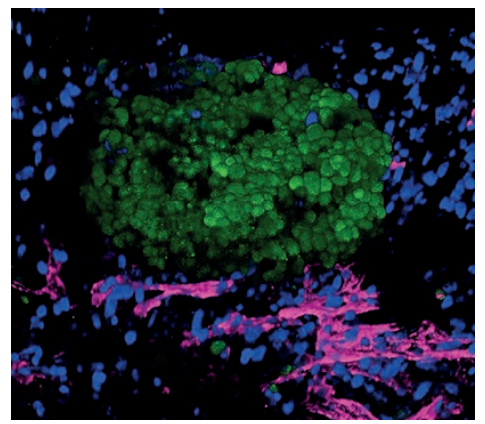

3D view of Msi reporter-positive pancreatic cancer cells (green) within a tumour mass. The vasculature is stained magenta, nuceli are blue. Taken from supplementary information from the original article.

platform to test new agents that might remove therapy-resistant cells more effectively. "Based on the exciting early-stage work with Msi ASOs, I hope there is a more concerted effort to target this pathway," she concludes.

lain Dickson

ORIGINAL ARTICLE Fox, R. G. et al. Image-based detection and targeting of therapy resistance in pancreatic adenocarcinoma. Nature http://dx.doi.org/10.1038/nature17988 (2016) 